\title{
Severe Hypercalcemia Mimicking Primary Hyperparathyroidism in the Context of Thyrotoxicosis: A Case Report
}

\author{
Abrar Gundroo $^{\mathrm{a}, \mathrm{b}}$, Karim Meeran ${ }^{\mathrm{a}}$
}

\begin{abstract}
We report a case of a 27-year-old woman who presented to our endocrinology clinic with two episodes of severe and symptomatic hypercalcemia associated with thyrotoxicosis in Graves' disease. The degree of hypercalcemia $(3.34 \mathrm{mmol} / \mathrm{L})$ is the most significant we have seen documented in the literature. On both occasions, we found that it was responsive to fluids and antithyroid medication. Unusually, we also noted that her severe hypercalcemia was associated with an unsuppressed parathyroid hormone level in the absence of demonstrable parathyroid disease. We conclude that thyrotoxicosis can be associated with a severe and symptomatic hypercalcemia and unsuppressed parathyroid hormone. Our case would suggest that it is important to screen for thyrotoxicosis in a new presentation of hypercalcemia, and that a diagnosis of primary hyperparathyroidism should not be made in the context of thyrotoxicosis.
\end{abstract}

Keywords: Hyperthyroid; Hypercalcemia; Parathyroid hormone; Primary hyperparathyroidism

\section{Introduction}

Hypercalcemia in thyrotoxicosis is usually a mild and asymptomatic finding and is associated with suppression of parathyroid hormone in the absence of concomitant hyperparathyroidism [1]. Here we describe an unusual case of a symptomatic and severe hypercalcemia with unsuppressed parathyroid function, in the context of thyrotoxicosis in Graves' disease, erroneously suggesting primary hyperparathyroidism.

\footnotetext{
Manuscript accepted for publication November 28, 2013

${ }^{\mathrm{a}}$ Department of Endocrinology, Imperial College Healthcare NHS

Trust, London, UK

${ }^{\mathrm{b}}$ Corresponding author: Abrar Gundroo, Department of Endocrinology, Charing Cross Hospital, Fulham Palace Road, London W6 8RF, UK.

Email: abrar@cantab.net
}

doi: http://dx.doi.org/10.4021/jem194w

\section{Case Report}

A 27-year-old woman presented to our endocrinology clinic with a 6-month history of thyrotoxic symptoms including weight loss of four stone, palpitations, tremor, fatigue and sweating. Over the last 6 weeks, she described a history of increasing polydipsia and polyuria, and had experienced persistent nausea and vomiting over the last 5 days. She was not taking any regular medications and she had a family history of Graves' disease in her mother and grandmother. On examination, she was tremulous with a pulse 100 beats/min and blood pressure 147/90 $\mathrm{mmHg}$. She was clinically dehydrated and a diffusely enlarged non-tender thyroid gland was palpable.

Her thyroid function tests demonstrated marked thyrotoxicosis, with free tetraiodothyronine (fT4) $69.1 \mathrm{pmol} / \mathrm{L}$ (normal range (NR) $9.0-26.0 \mathrm{pmol} / \mathrm{L}$ ), free triiodothyronine (fT3) $46.1 \mathrm{pmol} / \mathrm{L}$ (NR 2.5 - $5.7 \mathrm{pmol} / \mathrm{L}$ ) and thyroid stimulating hormone $<0.05 \mathrm{mU} / \mathrm{L}$ (NR $0.3-2.5 \mathrm{mU} / \mathrm{L}$ ). Bone profile revealed a pronounced hypercalcemia with serum adjusted calcium $3.34 \mathrm{mmol} / \mathrm{L}$ (NR 2.15 - $2.60 \mathrm{mmol} / \mathrm{L}$ ), phosphate $1.50 \mathrm{mmol} / \mathrm{L}(\mathrm{NR} 0.18-1.4 \mathrm{mmol} / \mathrm{L}$ ) and parathyroid hormone level $2.1 \mathrm{pmol} / \mathrm{L}$ (NR $1.1-6.8 \mathrm{pmol} / \mathrm{L}$ ). Urine calcium was $0.71 \mathrm{mmol} / \mathrm{L}$. Serum creatinine was $76 \mu \mathrm{mol} / \mathrm{L}$ (NR $50.0-120.0 \mu \mathrm{mol} / \mathrm{L}$ ). Investigations to determine an alternative cause of hypercalcemia were sought. Serum protein electrophoresis was normal, chest X-ray was clear and breast screening was unremarkable. Furthermore, there was no history of recent immobilization or exogenous vitamin D ingestion. Subsequent antibody tests and imaging confirmed a diagnosis of Graves' disease.

She was admitted to the endocrinology ward and treated with high-dose carbimazole, propranolol and intravenous fluids. She responded very well to treatment and investigations 1 week later showed normalized thyroid function (fT4 $16.0 \mathrm{pmol} / \mathrm{L}$, fT3 $4.2 \mathrm{pmol} / \mathrm{L}$ ) and reversal of hypercalcemia (serum adjusted calcium $2.10 \mathrm{mmol} / \mathrm{L}$ ). Repeat parathyroid hormone level at this time measured $2.5 \mathrm{pmol} / \mathrm{L}$.

On discharge, her compliance with carbimazole medication was poor and consequently she re-presented to clinic with thyrotoxicosis 4 months later. On this occasion, blood 


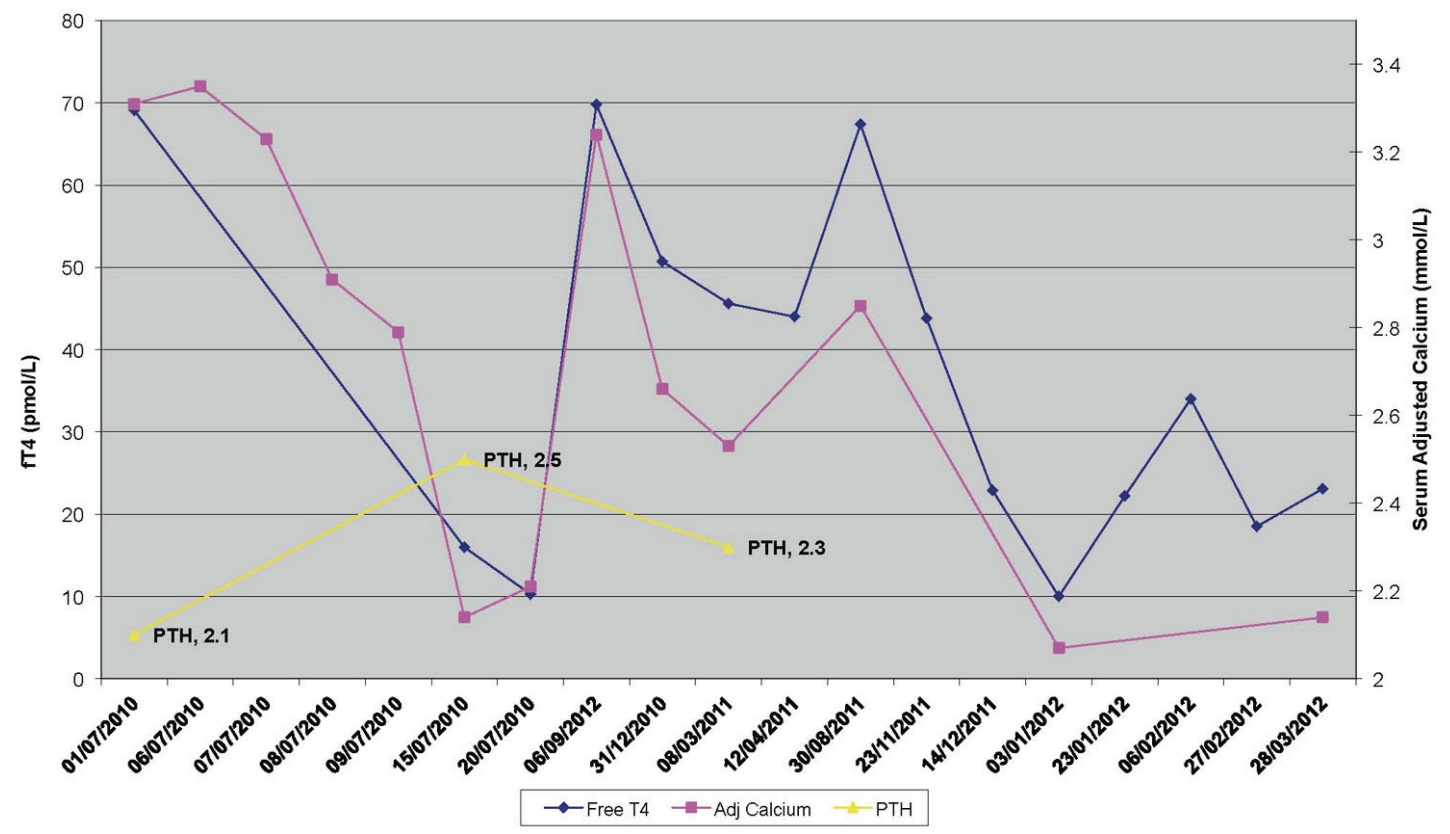

Figure 1. Serum adjusted calcium, parathyroid hormone (PTH) and fT4 levels since initial presentation.

tests again showed marked thyrotoxicosis (fT4 $69.8 \mathrm{pmol} / \mathrm{L}$, fT3 $46.1 \mathrm{pmol} / \mathrm{L}$ ) with associated hypercalcemia (serum adjusted calcium $3.24 \mathrm{mmol} / \mathrm{L}$ ). This again responded well to fluid and antithyroid treatment. Her serum calcium levels have subsequently remained stable and parathyroid hormone measurements 6 months after initial presentation were normal. Figure 1 shows her free T4, serum adjusted calcium and parathyroid hormone levels since initial presentation, demonstrating an association of thyrotoxicosis with hypercalcemia and normal parathyroid hormone levels.

\section{Discussion}

Hypercalcemia in hyperthyroidism is usually a mild and asymptomatic phenomenon. It is thought to be primarily mediated through the effects of thyroid hormone on bone resorption, particularly increased bone turnover as a consequence of fT3-induced osteoclast differentiation [2]. The most common cause of symptomatic hypercalcemia in the context of hyperthyroidism is concomitant primary hyperparathyroidism, which is a rare occurrence [3].

This case demonstrates two unusual features. Firstly, a severe and symptomatic hypercalcemia associated with thyrotoxicosis, demonstrated on two separate occasions. This degree of hypercalcemia $(3.34 \mathrm{mmol} / \mathrm{L})$ is the most significant we have seen documented in the literature [4-6]. It was clear from her presentation that she had symptoms of both hypercalcemia and thyrotoxicosis; however, this may not always be clear-cut, therefore it is important to consider hyper- thyroidism in patients with predominant symptoms of hypercalcemia. On both occasions, we found her elevated serum calcium was responsive to fluid rehydration and restoration of euthyroid status with antithyroid medication.

Unusually, we also noted that on initial presentation, her severe hypercalcemia was associated with an unsuppressed parathyroid hormone level, suggesting primary hyperparathyroidism. There are, however, a number of observations that would exclude this diagnosis. Firstly, we see a resolution of hypercalcemia following achievement of euthyroid status and adequate rehydration, on two separate occasions. In between these episodes, serum calcium and parathyroid hormones remain within normal range, and have subsequently remained normal. We also note a raised serum phosphate on initial presentation, which is most in keeping with increased tubular reabsorption of phosphate seen in hyperthyroidism [7]. Conversely one would expect a low serum phosphate in primary hyperparathyroidism as a result of decreased renal tubular reabsorption [8].

Our findings would suggest there is unsuppressed parathyroid hormone activity associated with thyrotoxicosis, without demonstrable parathyroid disease. It is plausible that this activity contributes to the severity of hypercalcemia seen in this case.

\section{Conclusion}

Thyrotoxicosis can be associated with severe and symptomatic hypercalcemia and unsuppressed parathyroid hormone, without demonstrable parathyroid disease. In this instance, 
hypercalcemia appears to be a reversible phenomenon responsive to fluid and antithyroid therapy. Our case would suggest that it is important to screen for thyrotoxicosis in a new presentation of hypercalcemia, and that a diagnosis of primary hyperparathyroidism should not be made in the context of thyrotoxicosis.

\section{Disclosure}

The author reports no conflicts of interest in this work.

\section{References}

1. Daly JG, Greenwood RM, Himsworth RL. Serum calcium concentration in hyperthyroidism at diagnosis and after treatment. Clin Endocrinol (Oxf). 1983;19(3):397404.

2. Begic-Karup S, Wagner B, Raber W, Schneider B, Hamwi A, Waldhausl W, Vierhapper H. Serum calcium in thyroid disease. Wien Klin Wochenschr. 2001;113(12):65-68.

3. Wagner B, Begic-Karup S, Raber W, Schneider B, Waldhausl W, Vierhapper H. Prevalence of primary hyperparathyroidism in 13387 patients with thyroid diseases, newly diagnosed by screening of serum calcium. Exp Clin Endocrinol Diabetes. 1999;107(7):457-461.

4. Richards AJ. Hypercalcaemia in thyrotoxicosis with and without hyperparathyroidism. Postgrad Med J. 1970;46(537):440-446.

5. Baxter JD, Bondy PK. Hypercalcemia of thyrotoxicosis. Ann Intern Med. 1966;65(3):429-442.

6. Parfitt AM, Dent CE. Hyperthyroidism and hypercalcaemia. Q J Med. 1970;39(154):171-187.

7. Auwerx J, Bouillon R. Mineral and bone metabolism in thyroid disease: a review. Q J Med. 1986;60(232):737752 .

8. Broulik PD, Pacovsky V. Renal tubular maximum reabsorptive capacity for phosphate in patients with primary hyperparathyroidism before and after operation. Exp Clin Endocrinol. 1986;87(1):43-47. 